\title{
Capacitance of Biconical Antennas in Magneto-Ionic Media; Elliptic Cone Capacitance
}

\author{
V. P. Pyati and H. Weil \\ Contribution From the Radio Astronomy Observatory, University of Michigan, Ann Arbor, Mich.
}

(Received August 4, 1964)

\begin{abstract}
Two antenna capacitance problems are solved in this paper. The motivating problem was to determine the capacitance of a biconical antenna of circular cross section immersed in a magneto-ionic medium. This is solved by reducing it to the secondary problem of determining the capacitance of a biconical antenna of elliptic cross section in an isotropic medium. The latter is solved using the sphero-conal coordinate system.
\end{abstract}

\section{Introduction}

Satellite and rocket borne experiments carried out in the ionosphere utilize antennas for various purposes in ways which require an understanding of the effects of the ionospheric environment on the antenna impedance. In particular, many experiments are operated at frequencies and altitudes such that the anisotropic properties of the ionosphere come strongly into play. Examples occur in the reception of cosmic noise in the topside of the ionosphere at frequencies near $1 \mathrm{Mc} / \mathrm{s}$ [Walsh et al., 1963] and in the use of impedance probes to measure ambient electron densities [Herman, 1964]. In terms of the well known ionospheric parameters

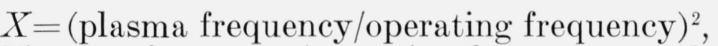

$Y=$ gyro frequency/operating frequency, and

$Z=$ collision frequency/operating frequency

it turns out that over a large part of the $X Y^{2}$ plane the capacitive part of the impedance is dominant. This capacitance can be thought of as made up approximately of parallel capacitances across the space charge sheath, i.e., from antenna surface to undisturbed plasma, and between the two parts of the antenna at different potentials. This latter capacitance has been computed in the literature by neglecting the modification of the embedding plasma due to the sheath [Herman, 1964; Kaiser 1962; and Balmain 1964]. Kaiser has considered a biconical antenna and Balmain a cylindrical one, while Herman's result is rough enough to require only a thin symmetrical antenna with no further shape details.

To carry out his analysis of the conical antenna Kaiser [1962] employed an approximate mathematical procedure whose accuracy is difficult to assess. The purpose of this work is to obtain an exact solution for the same physically simplified problem; that of a conical antenna in a plasma, neglecting the sheath. This clears the way for evaluating the effects of the sheath by removing the effects of the approximate mathematics. The latter are negligible when the antenna axis is oriented along the earth's magnetic field $H$, but become significant if the antenna orientation approaches $90^{\circ}$ to $H$. Both the present results evaluated for thin cones as well as Kaiser's and Balmain's [1964] results are all quite different from those of Herman. The primary reason is that Herman [1964] incorrectly assumed that the value of the integral over the antenna surface of the electric field vector $E$ is not different when the antenna is in free space from its value when the antenna is immersed in a plasma.

Interest in biconical antennas of circular cross section stems in part from the fact that a thin biconical antenna approximates a thin cylindrical antenna and is easier to treat mathematically. In an isotropic medium the capacitance per unit length (along a generator) of an unterminated biconical antenna has the pleasant property of being constant and is given by

$$
c=\frac{\pi \epsilon}{\left|\ln \tan \frac{\beta}{2}\right|} \approx \frac{\pi \epsilon}{\ln \left(\frac{2}{\beta}\right)}
$$

for small $\beta$. Here $\beta$ is the semiangle of the cone and $\epsilon$ is the dielectric constant. The capacitance of a biconical antenna of circular cross section but finite length $l$ measured along the generators is then simply

$$
C=c l
$$

if fringing is neglected.

The plan of this paper is to first work out the analogous formulas for the case of a cone of elliptical cross section. This is done in section 2. Then in section 3 the reduction of the capacitance problem for a biconical antenna of circular cross section in anisotropic plasma to the problem solved in section 2 is carried out. This is an application to the present 


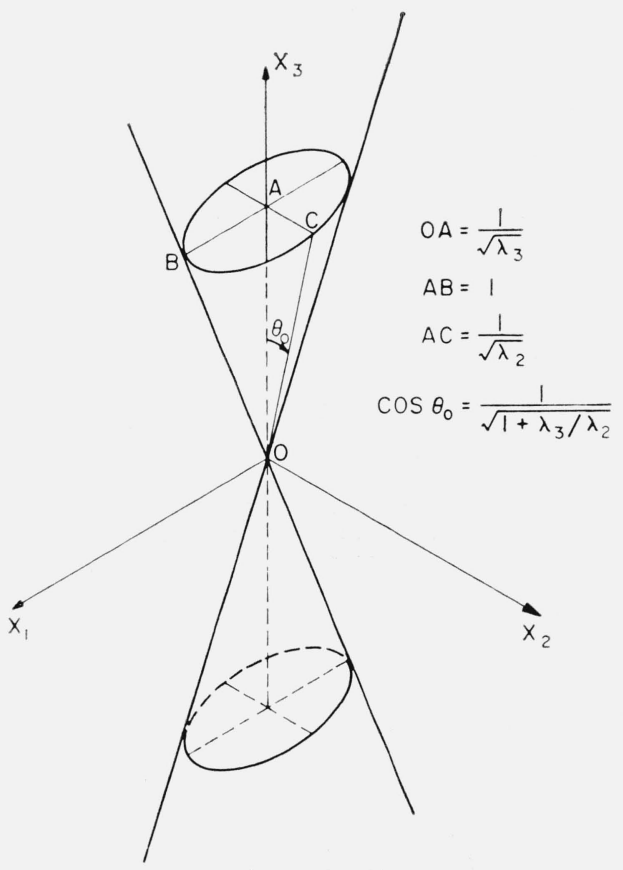

Figure 1. Elliptic cone in isotropic medium.

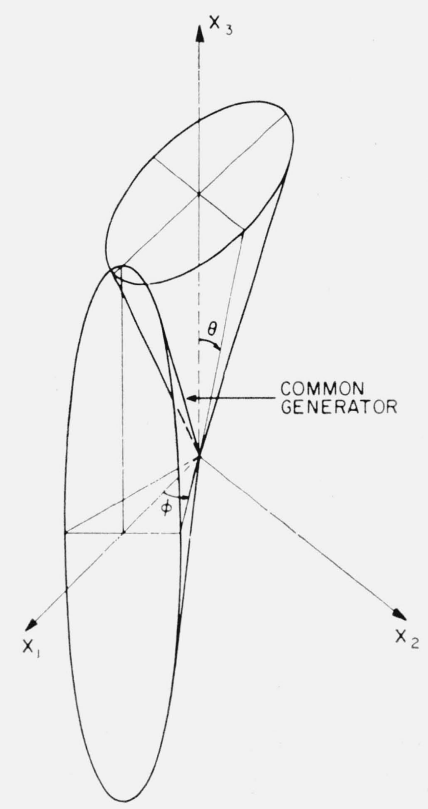

FIGURE 2. The sphero-conal coordinate system. problem of a procedure given in Landau and Lifsbitz [1960].

Since use is made in section 2 of the rather little known sphero-conal coordinate system, a brief discussion of the form of the coordinate surfaces and their analytic representation is included, largely following the work of Kraus and Levine [1961].

\section{Capacitance of Elliptic Cone in Isotropic Medium}

Consider the boundary value problem

$$
\frac{\partial^{2} V}{\partial X_{1}^{2}}+\frac{\partial^{2} V}{\partial X_{2}^{2}}+\frac{\partial^{2} V}{\partial X_{3}^{2}}=0
$$

and $V= \pm V_{0}$ on the surface of the elliptical cone

$$
X_{1}^{2}+\lambda_{2} X_{2}^{2}=\lambda_{3} X_{3}^{2}
$$

as shown in figure $1 ; \lambda_{2}, \lambda_{3}$ are positive and we assume $\lambda_{2}>1$

It is clear from the symmetry of the problem that the equipotentials are elliptic cones and the lines of force meridian lines. The natural coordinate system in this case is the sphero-conal system. Hobson [1931] and Kraus and Levine [1961] have given clear and concise treatments. The spheroconal system uses concentric spheres and two sets of elliptical cones as the three sets of mutually orthogonal surfaces. The concentric spheres are given by

$$
X_{1}^{2}+X_{2}^{2}+X_{3}^{2}=r^{2} .
$$

The two sets of cones are shown in figure 2 and are given by

$$
\begin{gathered}
\frac{X_{1}^{2}}{\mu^{2}}+\frac{X_{2}^{2}}{\mu^{2}-h^{2}}-\frac{X_{3}^{2}}{l^{2}-\mu^{2}}= \\
\frac{X_{1}^{2}}{\nu^{2}}-\frac{X_{2}^{2}}{h^{2}-\nu^{2}}-\frac{X_{3}^{2}}{l^{2}-\nu^{2}}=0 \quad 0 \leq \nu^{2} \leq h^{2} \leq \mu^{2} \leq l^{2}, \\
\quad l>h>0 .
\end{gathered}
$$

Here $l$ and $h$ are arbitrary constants and for each value of $\mu$, system (5) represents an ellipse for constant $X_{3}$ with its major axis in the $X_{1} X_{3}$ plane. Similarly for each value of $\nu$ system (6) represents an ellipse for constant $X_{1}$ with its major axis in the $X_{1} X_{3}$ plane. The relation between the $\left(X_{1}\right.$, $\left.X_{2}, X_{3}\right)$ and $(r, \mu, \nu)$ systems is given by

$$
\begin{gathered}
X_{1}=\frac{r \mu \nu}{h l} \quad X_{2}=\frac{r \sqrt{\left(\mu^{2}-h^{2}\right)\left(h^{2}-\nu^{2}\right)}}{h \sqrt{l^{2}-h^{2}}}, \\
X_{3}=\frac{r \sqrt{\left(l^{2}-\mu^{2}\right)\left(l^{2}-\nu^{2}\right)}}{l \sqrt{l^{2}-h^{2}}} .
\end{gathered}
$$

The cone of our problem given by (3) can be made 
to fit the notation of (5) if for convenience we take $\mu^{2}=1$ and equate coefficients to obtain

$$
h^{2}=\frac{\lambda_{2}-1}{\lambda_{2}} \text { and } l^{2}=\frac{\lambda_{3}+1}{\lambda_{3}} .
$$

The coordinates $(r, \mu, \nu)$ are still not very convenient for our purpose. Kraus and Levine [1961] have defined an equivalent set of polar coordinates $(r, \theta, \varphi)$ to replace $(r, \mu, \nu)$ with ranges of values

$$
0 \leq \theta \leq \pi, 0 \leq \varphi \leq 2 \pi, r \geq 0
$$

Here $\theta$ is the angle between the positive $X_{3}$ axis and the generator drawn through an end of the minor axis of the ellipse described by (5). Similarly, $\varphi$ is the angle between the positive $X_{1}$ axis and the generator drawn through an end of the minor axis described by (6). Figure 2 may be used as an aid to visualize these surfaces. The following relations can be established

where

$$
\begin{aligned}
\cos \varphi & =\frac{\nu}{h}, \cos \theta=\sqrt{\frac{l^{2}-\mu^{2}}{l^{2}-h^{2}}} \\
X_{1} & =r\left(1-k^{2} \cos ^{2} \theta\right)^{1 / 2} \cos \varphi, \\
X_{2} & =r \sin \theta \sin \varphi, \\
X_{3} & =r \cos \theta\left(1-k^{\prime 2} \cos ^{2} \varphi\right)^{1 / 2},
\end{aligned}
$$

$$
k^{\prime 2}=\frac{h^{2}}{l^{2}}=\frac{\tan ^{2} \epsilon \cos ^{2} \theta}{1+\tan ^{2} \epsilon \cos ^{2} \theta} \text { and } k^{2}=1-k^{\prime 2} .
$$

The semiangles of $\epsilon$ and $\epsilon^{\prime}$ subtended by the foci of the ellipses of systems (5) and (6) at the origin respectively are given by

$$
\tan \epsilon=\frac{h}{\left(l^{2}-h^{2}\right)^{1 / 2} \cos \theta}, \text { and } \tan \epsilon^{\prime}=\frac{\left(l^{2}-h^{2}\right)^{1 / 2}}{h \cos \varphi} .
$$

We summarize the salient geometrical features of the sphero-conal system. The surfaces $r=$ const. are spheres centered at the origin. The $\theta=$ const. surfaces are elliptic cones belonging to system (5). Similarly, $\varphi=$ const. surfaces are elliptic cones belonging to system (6). The equations of these surfaces can be found by eliminating $\mu, \nu$ in (5) and (6) with the help of (9). Two such intersecting surfaces are shown in figure 2. The line indicated as "common generator" is a line of intersection. This common generator traces out the elliptic cones of system (5) by rotating around the $X_{3}$ axis and when this generator lies in the $X_{2} X_{3}$ plane it makes an angle $\theta$ with the $X_{3}$ axis. This same generator traces out the elliptic cone of system (6) by rotating around the $X_{1}$ axis making an angle $\varphi$ with the $X_{1}$ axis when it lies in the $X_{1} X_{2}$ plane. Finally, when $k=1$, the system degenerates into the familiar spherical system in the following fashion. As $k \rightarrow 1$, the elliptic cones given by $\theta=$ const. become more and more circular. In the limit when $k=1, \epsilon=0$ implying that the foci have coalesced and we have cones of revolution.
As for the $\varphi=$ const. surfaces, as $k \rightarrow 1$, the major axis becomes longer and when $k=1, \epsilon^{\prime}=\frac{\pi}{2}$ meaning that the foci have moved out to infinity, and the elliptical cone degenerates into a plane.

In terms of these coordinates the cone of our problem is the surface $\theta=\theta_{0}$ where

$$
\left.\cos \theta_{0}=\left[1+\lambda_{3} / \lambda_{2}\right)\right]^{-1 / 2} .
$$

The Laplace and gradient operator are given by

$$
\begin{aligned}
& \nabla^{2}=\frac{1}{r^{2}} \frac{\partial}{\partial r}\left(r^{2} \frac{\partial}{\partial r}\right)+\frac{1}{r^{2}\left(k^{2} \sin ^{2} \theta+k^{\prime 2} \sin ^{2} \varphi\right)} \\
& {\left[\left(1-k^{2} \cos ^{2} \theta\right)^{1 / 2} \frac{\partial}{\partial \theta}\left\{\left(1-k^{2} \cos ^{2} \theta\right)^{1 / 2} \frac{\partial}{\partial \theta}\right\}\right.} \\
&+\left(1-k^{\prime 2} \cos ^{2} \theta\right)^{1 / 2} \frac{\partial}{\partial \varphi} \\
&\left.\left\{\left(1-k^{\prime 2} \cos ^{2} \varphi\right)^{1 / 2} \frac{\partial}{\partial \varphi}\right\}\right]
\end{aligned}
$$$$
\nabla=\mathbf{l}_{\mathbf{r}} \frac{\partial}{\partial r}+\mathbf{l}_{\theta} \frac{\left(1-k^{2} \cos ^{2} \theta\right)^{1 / 2}}{r\left(k^{2} \sin ^{2} \theta+k^{\prime 2} \sin ^{2} \varphi\right)^{1 / 2}} \frac{\partial}{\partial \theta}
$$$$
+\mathbf{l} \frac{\left(1-k^{\prime 2} \cos ^{2} \varphi\right)^{1 / 2}}{r\left(k^{2} \sin ^{2} \theta+k^{\prime 2} \sin ^{2} \varphi\right)^{1 / 2}} \frac{\partial}{\partial \varphi} .
$$

Considering the Laplace equation we note that in the present problem there is no variation of $V$ with respect to $r$ and $\varphi$. Integrating we get

$$
V(\theta)=A \int_{\theta_{0}}^{\theta}\left(1-k^{2} \cos ^{2} \theta\right)^{-1 / 2} d \theta+B .
$$

The constants $A$ and $B$ can be determined from the boundary conditions

$$
V\left(\theta_{0}\right)=V_{0}, V\left(\pi-\theta_{0}\right)=-V_{0} .
$$

Solving for $A$ and $B$ we get

$$
V(\theta)=V_{0} \frac{F\left(\frac{\pi}{2}-\theta, k\right)}{F\left(\frac{\pi}{2}-\theta_{0}, k\right)} .
$$

Here $F$ is the incomplete elliptic integral of the first kind given by

$$
F(\theta, k)=\int_{0}^{\theta}\left(1-k^{2} \sin ^{2} \varphi\right)^{-1 / 2} d \varphi, \quad k^{2}<1 .
$$

The surface charge density is obtained with the help of the gradient operator (12) applied to the potential function (15). For this problem $D=\mathbf{D} \cdot \mathbf{1}_{\theta}=\sigma$ and

$$
\sigma=\frac{1}{r} \frac{\epsilon V_{0}}{F\left(\frac{\pi}{2}-\theta_{0}, k\right)} \cdot\left(k^{2} \sin ^{2} \theta_{0}+k^{2} \sin ^{2} \varphi\right)^{-1 / 2} .
$$


It is seen that, in spite of the $\varphi$ independence of $V, \sigma$ is $\varphi$ dependent. The elemental area is given by

$$
d \Omega=\frac{r\left(k^{2} \sin ^{2} \theta_{0}+k^{\prime 2} \sin ^{2} \varphi\right)^{1 / 2}}{\left(1-k^{\prime 2} \cos ^{2} \varphi\right)^{1 / 2}} d r d \varphi
$$

Integrating $D$ over $0 \leq \varphi \leq 2 \pi, 0 \leq r \leq 1$ and dividing by $2 V_{0}$, yields the capacitance $c$ per unit length measured in the radial direction

$$
\begin{aligned}
c & =\frac{\epsilon}{2 F\left(\frac{\pi}{2}-\theta_{0}, k\right)} \int_{0}^{2 \pi}\left(1-k^{\prime 2} \cos ^{2} \varphi\right)^{-1 / 2} d \varphi \\
& =\frac{2 K^{\prime} \epsilon}{F\left(\frac{\pi}{2}-\theta_{0}, k\right)} .
\end{aligned}
$$

In the above result $K^{\prime}$ is the complete elliptic integral of the first kind of modulus $k^{\prime}$. Here a cone of unit length is interpreted as that portion of the cone enclosed by a sphere of unit radius. For the coordinate system we are using, cones of this description are suitable because integration in $r$ will be particularly simple.

\section{Capacitance Problems in Anisotropic Media}

Assuming the static superposed magnetic field to be in the $z$ direction, the dielectric tensor (of a magneto-ionic medium) has the form

$$
\overleftrightarrow{K}=\left[\begin{array}{lrr}
\alpha_{1} & -\alpha_{2} & 0 \\
\alpha_{2} & \alpha_{1} & 0 \\
0 & 0 & \alpha_{3}
\end{array}\right] .
$$

In particular when collisions are neglected

$$
\alpha_{1}=1-\frac{X}{1-Y^{2}}, \alpha_{2}=\frac{i X Y}{1-Y^{2}} \text { and } \alpha_{3}=1-X,
$$

where $X=\frac{\omega_{p}^{2}}{\omega^{2}}, Y=\frac{\omega_{H}}{\omega}, \omega_{p}$ and $\omega_{H}$ are plasma and gyrofrequencies.

The capacitance will be determined using a quasistatics approximation in which electric field $\mathbf{E}$ is derivable from a gradient $V$ (which is valid, strictly speaking, at zero frequency), but using the dielectric tensor components evaluated at the frequency of interest. This procedure is justifiable, since capacitance is essentially a near field quantity, so long as the dimensions of the antenna are not large compared to the wavelengths of the radiation. This will be the case in most regions of the $X-Y^{2}$ plane shown in figure 3 . However, for certain angles $\theta$ between the propagation vector and the superposed magnetic field the index of refraction for the ordinary mode becomes very large (infinite in the collision free case) in regions 7 and 8 and similarly for the

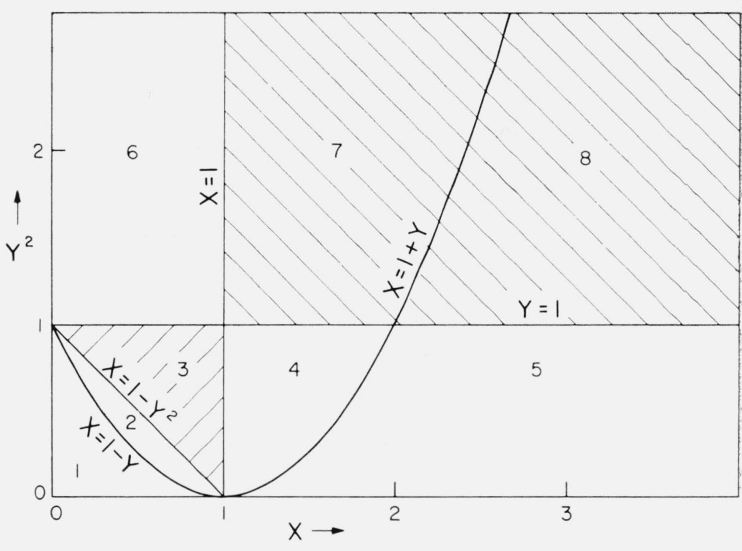

Figure 3. The $\mathrm{X}-\mathrm{Y}^{2}$ plane.

extraordinary mode in region 3. The associated wavelengths are then small and near zone or quasistatics approximations become quite questionable. Furthermore, the radiation resistance in regions 3, 7, and 8 becomes very large [Weil and Walsh, 1964] and from this point of view one also sees that a quasistatics procedure (which cannot yield radiation) cannot be expected to be valid. This will be discussed further in section 4 .

The reduction of the capacitance determination for objects in a medium described by a Hermitian $\overleftrightarrow{K}$ to a related capacitance problem in an isotropic medium is demonstrated in Landau and Lifshitz [1960] beginning with the field due to a point charge. One has

and

$$
\nabla \cdot \mathbf{D}=e \delta(x) \delta(y) \delta(z)
$$

$$
\mathbf{D}=\overleftrightarrow{\epsilon_{0}} \overleftrightarrow{K} \cdot \mathbf{E}
$$

Defining an electrostatic potential $\mathbf{E}=-\nabla V$ and substituting in the above equations, we note that the mixed derivatives cancel on account of the Hermitian nature of the tensor and the equation for the potential $V$ becomes

$$
\frac{\partial^{2} V}{\partial x^{2}}+\frac{\partial^{2} V}{\partial y^{2}}+q^{2} \frac{\partial^{2} V}{\partial z^{2}}=-\frac{e}{\epsilon_{0} \alpha_{1}} \delta(x) \delta(y) \delta(z)
$$

where

The transformation

$$
q^{2}=\frac{\alpha_{3}}{\alpha_{1}}
$$

$$
x^{\prime}=x, y^{\prime}=y, z^{\prime}=\frac{z}{q}
$$

reduces (22) to a conventional Poisson's equation

$$
\frac{\partial^{2} V}{\partial x^{\prime 2}}+\frac{\partial^{2} V}{\partial y^{\prime 2}}+\frac{\partial^{2} V}{\partial z^{\prime 2}}=-\frac{e}{\epsilon_{0} \alpha_{1} q} \delta\left(x^{\prime}\right) \delta\left(y^{\prime}\right) \delta\left(z^{\prime}\right)
$$

for a point charge of strength $e / q \alpha_{1}$ in vacuum 
(primed space). Thus

$$
V=\frac{1}{4 \pi \epsilon_{0}}\left(\frac{e}{q \alpha_{1}}\right)\left[x^{2}+y^{2}+\frac{z^{2}}{q^{2}}\right]^{-1 / 2} .
$$

The above concept is applicable in capacitance problems since the surface charge densities must scale in the same way as individual charges.

The capacitance $C$ of an object in an anisotropic medium may now be determined as follows. First, one determines the capacitance $C^{\prime}$ in vacuum of the related object obtained by applying transformation (23). If one assumes that changing from unprimed to the primed system and vice versa the potential of the body does not change then the total charge residing on the surface must change in the same manner as for a point charge. Based on this we have

$$
C=q \alpha_{1} C^{\prime}
$$

The determination of $C^{\prime}$ is the secondary problem referred to in the abstract.

The procedure employed by Kaiser [1962] was to transform the potential function obtained in the primed system back to the unprimed system; then to obtain the total charge by integrating the normal component of $\mathbf{D}$ over the surface of the original antenna. Such a procedure has some drawbacks in general. First, the recovery of the potential function in terms of the unprimed system can be quite formidable. Second, $\mathbf{D}$ and $\mathbf{E}$ will not be parallel in the unprimed system thus introducing further complications. Both these difficulties are avoided by using (26).

We now revert to our specific problem. Consider a biconical antenna of semiangle $\beta$ whose axis makes an angle $\alpha$ with the magnetic field and lies in the $y z$ plane as shown in figure 4 .

The problem is to solve

$$
\frac{\partial^{2} V}{\partial x^{2}}+\frac{\partial^{2} V}{\partial y^{2}}+q^{2} \frac{\partial^{2} V}{\partial z^{2}}=0
$$

subject to the condition that $V= \pm V_{0}$ on the surface of the cone given by

$$
x^{2}+(-z \sin \alpha+y \cos \alpha)^{2}=(z \cos \alpha+y \sin \alpha)^{2} \tan ^{2} \beta \text {. }
$$

Applying the transformation (23) to (27) and (28), we get the following related problem in vacuum

$$
\frac{\partial^{2} V}{\partial x^{\prime 2}}+\frac{\partial^{2} V}{\partial y^{\prime 2}}+\frac{\partial^{2} V}{\partial z^{\prime 2}}=0
$$

and $V= \pm V_{0}$ on the elliptic cone

$x^{\prime 2}+\left(1-\sin ^{2} \alpha \sec ^{2} \beta\right) y^{\prime 2}+q^{2}\left(1-\cos ^{2} \alpha \sec ^{2} \beta\right) z^{\prime 2}$

$$
-2\left(q \sin \alpha \cos \alpha \sec ^{2} \beta\right) y^{\prime} z^{\prime}=0 .
$$

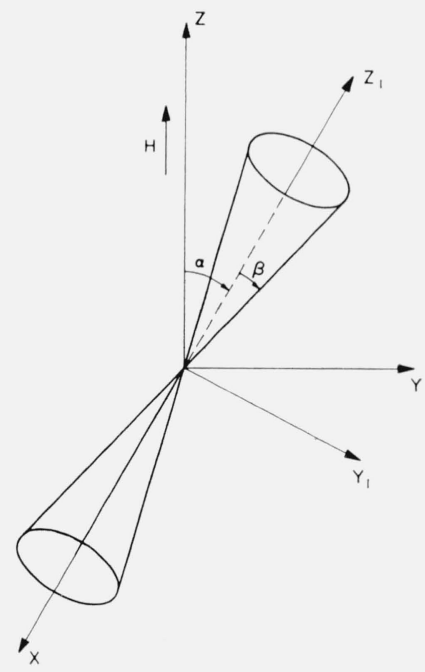

Figure 4. Biconical antenna in magneto-ionic medium.

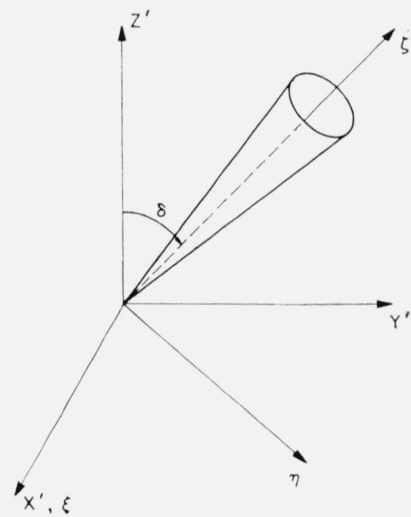

FiguRE 5. Elliptic cone of related problem given by (31).

First, we must reduce (30) to the normal form (3) by the usual diagonalization process. Defining a new coordinate system $\xi, \eta, \xi$ rotated through an angle $\delta$ from $x^{\prime}, y^{\prime}, z^{\prime}$, as shown in figure 5 , we have for the equation of the elliptic cone

$$
\xi^{2}+\lambda_{2} \eta^{2}=\lambda_{3} \zeta^{2}
$$

where

$$
\begin{aligned}
& \lambda_{2}, \lambda_{3}= \pm \frac{\left(1+q^{2}\right)-\sec ^{2} \beta\left(\sin ^{2} \alpha+q^{2} \cos ^{2} \alpha\right)}{2} \\
& +\frac{\left\{\left[\left(1+q^{2}\right)-\sec ^{2} \beta\left(\sin ^{2} \alpha+q^{2} \cos ^{2} \alpha\right)\right]^{2}+4 q^{2} \tan ^{2} \beta\right\}^{1 / 2}}{2}
\end{aligned}
$$

$\lambda_{2}$ goes with the upper and $\lambda_{3}$ goes with the lower sign. If $\lambda_{2}>1$, the elliptic cone (31) has the same orientation as that shown in figure 1 if we associate $(\xi, \eta, \zeta)$ with $\left(x_{1}, x_{2}, x_{3}\right)$. One can then determine $\theta_{0}$ using (10). On the other hand if $\lambda_{2}<1$, we see from (8) and (9) that $k^{2}>1$ and $k^{\prime 2}$ is negative which is not permissible. This difficulty can be 
overcome by rotating the $\xi, \eta$ coordinates through $90^{\circ}$ but keeping $\zeta$ fixed. Replacing $\xi$ by $-\eta_{1}$ and $\eta$ by $\xi_{1}$ in (31) we get

where

$$
\xi_{1}^{2}+\lambda_{2}^{\prime} \eta_{1}^{2}=\lambda_{3}^{\prime} \zeta^{2}
$$

$$
\lambda_{2}^{\prime}=\frac{1}{\lambda_{2}} \text { and } \lambda_{3}^{\prime}=\frac{\lambda_{3}}{\lambda_{2}}
$$

Now (33) is in the desired form. Using (10)

$$
\begin{aligned}
\cos \theta_{0} & =\left[1+\left(\frac{\lambda_{3}^{\prime}}{\lambda_{2}^{\prime}}\right)\right]^{-1 / 2} \\
& =\left(1+\lambda_{3}\right)^{-1 / 2}
\end{aligned}
$$

At this point it may be pointed out that part of the mathematical approximation of Kaiser [1962] lies in using, instead of the conal equipotential boundaries which correctly appear in the related problem, an approximate set of elliptic cylinder equipotentials. In the present analysis the correct boundaries are maintained. Approximations are put in only at the end in the final formula for capacitance to facilitate numerical computations for thin cones.

Before we apply the results of section 2 to this problem, we need an expression for the length of the elliptic cone. Let us assume that the radial length of the cone in figure 4 is unity. The coordinates $\left(x_{0}, y_{0}, z_{0}\right)$ of the tip of a generator are

$$
\begin{aligned}
& x_{0}=\sin \beta \cos \varphi \\
& y_{0}=\sin \alpha \cos \beta+\cos \alpha \sin \beta \sin \varphi \\
& z_{0}=\cos \alpha \cos \beta-\sin \alpha \sin \varphi \sin \beta
\end{aligned}
$$

where $\varphi$ is the azimuthal angle measured around the axis of symmetry $z_{1}$ (this $\varphi$ is different from the $\varphi$ of sphero-conal system) and varies from $0-2 \pi$. If $\left(x_{0}^{\prime}, y_{0}^{\prime}, z_{0}^{\prime}\right)$ be the tip of a generator of the elliptic cone then its length is given by

$$
\begin{aligned}
r_{0}^{\prime} & =\left(x_{0}^{\prime 2}+y_{0}^{\prime 2}+z_{0}^{\prime 2}\right)^{1 / 2} \\
& =\left(x_{0}^{2}+y_{0}^{2}+\frac{z_{0}^{2}}{q^{2}}\right)^{1 / 2} \text { using }(23) \\
& =\left[1-\frac{q^{2}-1}{q^{2}}(\cos \alpha \cos \beta-\sin \alpha \sin \beta \sin \varphi)^{2}\right]^{1 / 2} \\
& 0 \leq \varphi \leq 2 \pi .
\end{aligned}
$$

We note that the generators of the elliptic cone are of different lengths except when $\alpha=0$. For other angles of inclination, we will use an average value defined by

$$
\left\langle r_{0}^{\prime}\right\rangle=\frac{1}{2 \pi} \int_{0}^{2 \pi} r_{0}^{\prime}(\varphi) d \varphi .
$$

The above will lead to elliptic integrals. For small cone angles $\cos \beta \approx 1$ and ignoring the terms con- taining $\sin \beta$ we get

$$
\left\langle r_{0}^{\prime}\right\rangle \approx\left(\sin ^{2} \alpha+\frac{\cos ^{2} \alpha}{q^{2}}\right)^{1 / 2} .
$$

In this small angle case $\left\langle r_{0}^{\prime}\right\rangle$ is just the length of the cone axis.

The following special cases will now be considered.

Case (1): Axis of cone coinciding with the direction of the magnetic field. Here

$$
\begin{aligned}
& \alpha=0, \lambda_{2}=1, \lambda_{3}= q^{2} \tan ^{2} \beta, \\
& \theta_{0}=\tan ^{-1}(q \tan \beta), k=1, k^{\prime}=0 \\
& r_{0}^{\prime}=\left(\sin ^{2} \beta+\frac{\cos ^{2} \beta}{q^{2}}\right)^{1 / 2} .
\end{aligned}
$$

Using (19) for the capacitance per unit length of an elliptic cone in vacuum and the scale factor (26) we get

$$
c=\frac{\pi \epsilon_{0} \alpha_{1}\left(q^{2} \sin ^{2} \beta+\cos ^{2} \beta\right)^{1 / 2}}{\left|\ln \left[\tan \left(\frac{\theta_{0}}{2}\right)\right]\right|} .
$$

Using trigonometrical relations we get

$$
\tan \left(\frac{\theta_{0}}{2}\right)=q^{-1}\left\{\left(\cot ^{2} \beta+q^{2}\right)^{1 / 2}+\cot \beta\right\} .
$$

For this special case the exact result has also been obtained by Kaiser [1962]. For small values of $\beta$ and $q$ the above formula becomes

$$
c=\frac{\pi \epsilon_{0} \alpha_{1}}{\ln \left(\frac{2}{q \beta}\right)} .
$$

Case (2): Axis of cone perpendicular to magnetic field. Here $\alpha=90^{\circ}, \lambda_{2}=q^{2}, \lambda_{3}=\tan ^{2} \beta$ and $\left\langle r_{0}^{\prime}\right\rangle \approx 1$.

Here we have two subcases $q^{2}>1$ and $q^{2}<1$. If $q^{2}>1$

$$
\tan \theta_{0}=\frac{\tan \beta}{q}
$$

and

$$
c=\frac{2 K^{\prime} \epsilon_{0} \alpha_{1} q}{F\left(\frac{\pi}{2}-\theta_{0}, k\right)}
$$

with

$$
k^{2}=\cos ^{2} \beta+\frac{\sin ^{2} \beta}{q^{2}}, k^{\prime 2}=\left(\sin ^{2} \beta-\frac{\sin ^{2} \beta}{q^{2}}\right) .
$$

For small $\beta$ this becomes

$$
c=\frac{\pi \epsilon_{0} \sqrt{\alpha_{1} \alpha_{3}}}{\ln \left(\frac{2 q}{\beta}\right)} .
$$

If $q^{2}<1$, the rotation of coordinates discussed following (32) is used.

$$
\tan \theta_{0}=\tan \beta
$$


and

$$
c=\frac{2 K^{\prime} \epsilon_{0} \alpha_{1} q}{F\left(\frac{\pi}{2}-\beta, k\right)}
$$

with

$$
k^{2}=\frac{\sec ^{2} \beta}{1+\frac{\tan ^{2} \beta}{q^{2}}}, k^{\prime 2}=\frac{\tan ^{2} \beta\left(\frac{1}{q^{2}}-1\right)}{1+\frac{\tan ^{2} \beta}{q^{2}}} .
$$

For small $\beta$ this becomes

$$
c=\frac{\pi \epsilon_{0} \sqrt{\alpha_{1} \alpha_{3}}}{\ln \left(\frac{2}{\beta}\right)} .
$$

As against these Kaiser [1962] obtains

$$
c=\frac{\pi \epsilon_{0} \sqrt{\alpha_{1} \alpha_{3}}}{\ln \left[\frac{4 q}{\beta(1+q)}\right]} .
$$

Case (3): Axis of cone making intermediate angles with magnetic field.

$$
\left\langle r_{0}^{\prime}\right\rangle \approx\left(\sin ^{2} \alpha+\frac{\cos ^{2} \alpha}{q^{2}}\right)^{1 / 2}
$$

so that

$$
c=\frac{2 K^{\prime} \epsilon_{0} \alpha_{3}}{q F\left(\frac{\pi}{2}-\theta_{0}, k\right)}\left(\sin ^{2} \alpha+\frac{\cos ^{2} \alpha}{q^{2}}\right)^{1 / 2} .
$$

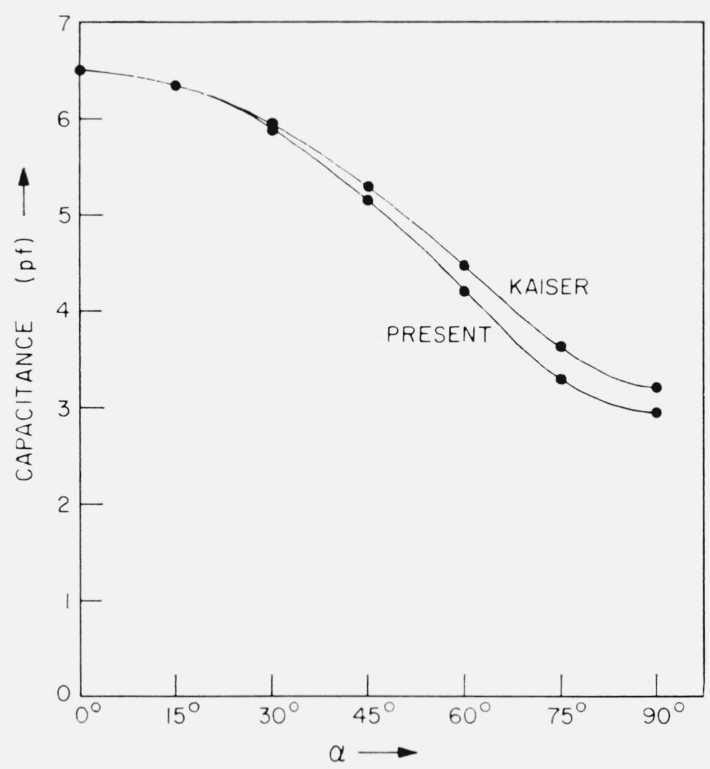

Figure 6. Capacitance as a function of inclination $\alpha$. $X=0.835, Y^{2}=3.33, \beta=1^{\circ}$.

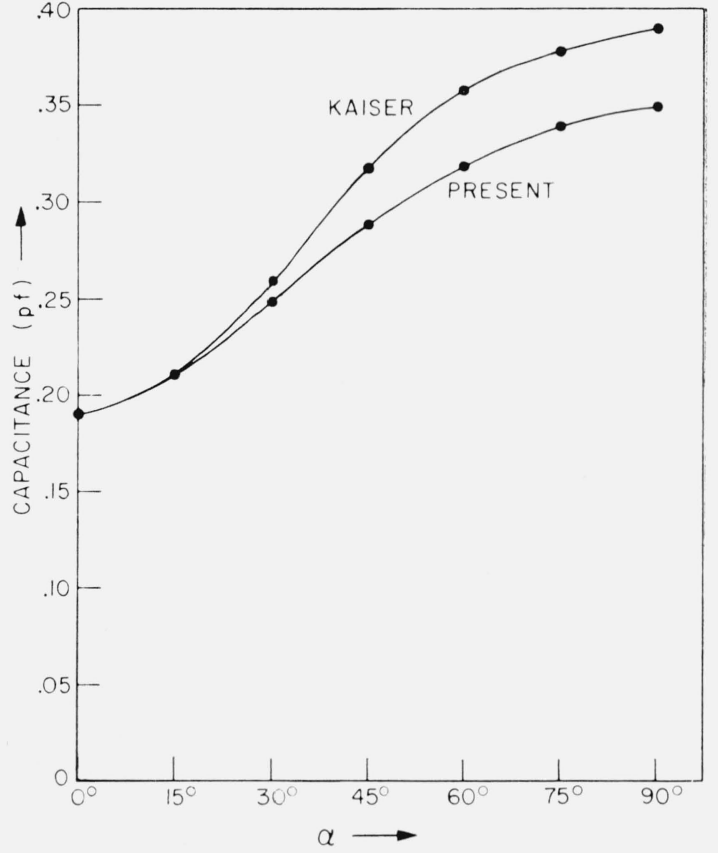

FiguRE 7. Capacitance as a function of inclination $\alpha$. $X=0.784, Y^{2}=0.196, \beta=1^{\circ}$.

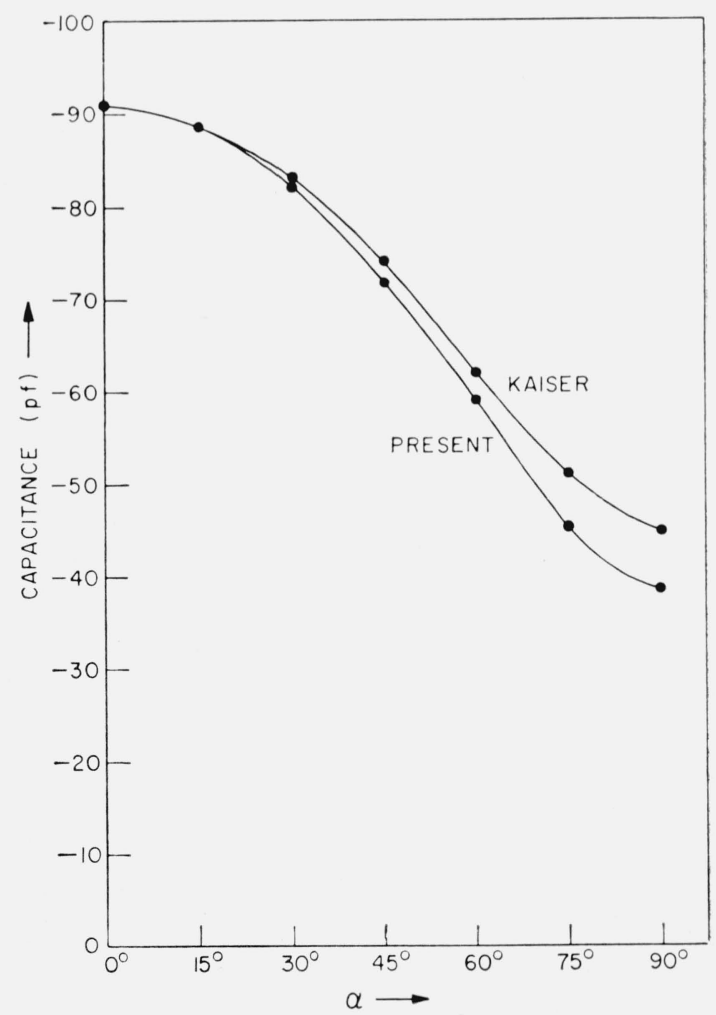

FIguRE 8. Capacitance as a function of inclination $\alpha$. $X=3.33, Y^{2}=0.835, \beta=1^{\circ}$. 


\section{Numerical Results and Discussion}

In practical situations where only cones of small angle are of interest the following approximations are valid

$$
k^{2} \rightarrow 1 \text { and } k^{\prime 2} \rightarrow 0
$$

so that

$$
K^{\prime} \approx \frac{\pi}{2}
$$

and

$$
F\left(\frac{\pi}{2}-\theta_{0}, k\right) \approx\left|\ln \tan \left(\frac{\theta_{0}}{2}\right)\right| .
$$

The resulting simplified capacitance formula

$$
c=\frac{\pi \epsilon_{0} \alpha_{3}}{q \ln \cot \left(\frac{\theta_{0}}{2}\right)}\left(\sin ^{2} \alpha+\frac{\cos ^{2} \alpha}{q^{2}}\right)^{1 / 2}
$$

will be utilized in numerical calculations. It must be noted that a further approximation of setting $\theta_{0}=\beta$ is not valid. This follows by considering (39) and (42). In the former $\theta_{0}=\tan ^{-1}(q \tan \beta)$ and in the latter $\theta_{0}=\tan ^{-1}\left(\frac{\tan \beta}{q}\right)$. Since these values depend on $q$, one cannot set $\theta_{0} \approx \beta$.

As we propose to compare present results with those of Kaiser's [1962], his formula is given below for convenience

$$
c=\frac{\pi \epsilon_{0} \alpha_{3}\left(\sin ^{2} \alpha+\frac{\cos ^{2} \alpha}{q^{2}}\right)^{1 / 2}}{q \ln \left\{\frac{4\left(q^{2} \sin ^{2} \alpha+\cos ^{2} \alpha\right)}{q \beta\left[1+\left(\cos ^{2} \alpha+q^{2} \sin ^{2} \alpha\right)^{1 / 2}\right]}\right\}} .
$$

In evaluating numerical results, it is necessary to know the ranges of values of $q^{2}$ for which formula (49) is valid. It may be recalled that we were required to solve the following partial differential equation

$$
\frac{\partial^{2} V}{\partial x^{2}}+\frac{\partial^{2} V}{\partial y^{2}}+q^{2} \frac{\partial^{2} V}{\partial z^{2}}=0
$$

with Dirichlet conditions. In a classical sense we have a well posed problem provided (27) is elliptic, i.e., $q^{2}>0$. In the primed space we have simply to solve Laplace's equation with $V$ given on real boundaries. To take into account complex values of $q^{2}$ corresponding to nonnegligible electron-neutral collisions in the plasma one can analytically continue the above expressions for $c$, valid for real $q^{2}$, into the complex $q^{2}$ plane, avoiding the necessary branch cuts. However, the physical meaning of the results becomes doubtful as one nears the negative real axis where $q^{2}<0$ and the equation in fact becomes hyperbolic. 'This is because $q^{2}<0$ corresponds to the collision free case for $X$ and $Y^{2}$ combinations which lie within regions 3 and 7 of figure 3 . In these regions, as previously pointed out, because of the short wavelengths in certain propagation directions, near zone (i.e., quasi-static) approximations are not valid. In addition, antenna impedance depends intimately on the current distributions and these are to be expected to be highly modified in regions 3 and 7 from those of an electrically short antenna.

For these reasons we would therefore not attribute physical significance to the result obtained by letting $q^{2}$ be negative, namely that there is an imaginary part in $c$ corresponding to an apparent radiation resistance and real loss of energy from the antenna to the collisionless plasma in regions 3 and 7 , in addition to the radiation loss which is found by using the full Maxwell equations.

In accord with the above remarks we will consider only regions of $q^{2}>0$ for numerical results. Representative values are shown graphically in figures 6 to 8 corresponding to regions 6,1 , and 5 , respectively. In region $5, X>1$, consequently capacitance is negative meaning that the antenna will be inductive. From the computed results we see that Kaiser's approximation is in agreement with the present results only for angles of inclination $\alpha$ around zero and deviates for increasing angles of inclination, reaching a 20 percent difference in some cases.

\section{References}

Balmain, K. G. (1964), The impedance of a short dipole antenna in a magneto plasma, IEEE Trans. Ant. Prop. AP-12, 605-617.

Herman, J. R. (1964), A method of determining D-region electron density profile utilizing a capacitive impedance rocket probe, J. Geophys. Res. 69, No. 11, 2329-2336.

Hobson, E. W. (1931), Spherical and ellipsoidal harmonics (Cambridge University Press).

Kaiser, T. R. (1962), The admittance of an electric dipole in a magneto-ionic environment, Planet Space Sci. 9, 639-657.

Kraus, L, and L. M. Levine (1961), Diffraction by an elliptic cone, Comm. Pure and Appl. Math. XIV, 49-69.

Landau, L. D., and E. M. Lifshitz (1960), Electrodynamies of continuous media (Addison-Wesley Pub. Co., Inc., Reading, Mass.).

Walsh, D., F. T. Haddock, and H. F. Schulte (June 1963), Cosmic radio intensities at 1.225 and $2.0 \mathrm{Mc} / \mathrm{s}$ measured up to an altitude of 1700 kilometers, Space Research IV, Proc. 4th International Space Science Symposium, Warsaw, Poland.

Weil, H., and D. Walsh (1964), Radiation resistance of an electric dipole in a magneto-ionic medium, IEEE Trans. Ant. Prop. AP-12, 297-304.

(Paper 69D2-466) 